\title{
T-fasteners for Preoperative Localization of Pulmonary Nodules
}

\begin{abstract}
Keywords: Pulmonary nodule; Preoperative localization; T-fastener Abstract

A 19 year-old male who presented with a persistent cough for three years was found to have multiple pulmonary nodules on imaging. A tissue diagnosis was necessary to determine the etiology of these nodules. Wedge resection of a peripheral nodule via video-assisted thoracoscopic surgery was planned. Pre-operative localization of this nodule was accomplished using a novel method of cone beam computed tomography and fluoroscopy where in a T-fastener and methylene blue were inserted. This method was well tolerated by the patient, permitted easier transport and positioning of the patient preoperatively, and allowed for clear identification of the nodule intraoperatively. In addition, the T-fastener is theoretically less traumatic to the lung than a traditional hook wire, and therefore less likely to cause a pneumothorax preoperatively. T-fasteners have been used extensively in the placement of gastrostomy tubes and this novel use of T-fasteners may be superior to current methods of preoperative pulmonary nodule localization.
\end{abstract}

\section{Introduction}

\section{Case presentation}

A 19 year-old male with a history of asthma presented with a 2-3 week history of persistent cough, $8 \%$ weight loss, fatigue and hypertension. A CT of his chest was performed showing interstitial reticular and nodular air space opacifications in the bases and lingula. Pulmonary function testing showed decreased pulmonary function with mild desaturations and an echocardiogram was normal. A flexible bronchoscopy with bronchoalveolar lavage (BAL) was performed and showed mild tracheomalacia and tongue base collapse. The BAL contained 400 nucleated cells per microliter, with a predominance of macrophages (83\%), lipid content of questionable significance, and few neutrophils (Figures 1A and 1B). Cultures from the BAL grew only rare Streptococcus viridans and rare coagulase negative Staphylococcus which was consistent with oral flora. He was started on tiotropium and initially had improvement in his symptoms.

One year later, the patient's weight stabilized but he continued to have a productive cough, now with wheezing and symptomatic desaturations. Repeat flexible bronchoscopy showed erythematous lower airway mucosa, which was associated with thick white/ yellow mucus. The BAL had a total nucleated cell count of 4000 per microliter with a predominance of neutrophils (84\%). A CT of the chest was then performed and demonstrated ground glass opacities and nodules in both lungs with predominance in the right lower lobe of the lung (Figure 2). The patient's presentation and imaging were concerning for an infectious bronchiolitis and a tissue diagnosis was required. VATS with preoperative nodule identification was scheduled for two weeks later.

\section{Journal of}

Pediatrics \& Child Care

\section{Shannon M Koehler ${ }^{1 *}$, Nathan Heinzerling ${ }^{1}$, David C Moe ${ }^{2,4}$, David C Gregg ${ }^{2,4}$, Sara Szabo ${ }^{3,4}$, James Southern ${ }^{4}$ and Amy J Wagner ${ }^{1,4}$}

${ }^{1}$ Pediatric Surgery Department, Children's Hospital of Wisconsin, 999 N 92nd Street, Suite 320, Milwaukee, WI 53226, USA

${ }^{2}$ Radiology Department, Children's Hospital of Wisconsin, $9000 \mathrm{~W}$. Wisconsin Avenue, PO Box 1997, Milwaukee, WI 53201-1997, USA ${ }^{3}$ Pathology Department, Children's Hospital of Wisconsin, $9200 \mathrm{~W}$. Wisconsin Avenue, Milwaukee, WI 53226-3522, USA

${ }^{4}$ Medical College of Wisconsin, Milwaukee, WI 53226, USA

*Address for Correspondence:

Shannon Koehler, MD, PhD, Pediatric Surgery Department, Children's Hospital of Wisconsin, 999 N 92nd Street, Suite 320, Milwaukee, WI 53226, USA, Tel: (414) 955-2243; E-mail: skoehler@chw.org

Submission: 01 December, 2015

Accepted: 05 January, 2016

Published: 11 January, 2016

Copyright: ( $) 2016$ Koehler SM. This is an open access article distributed under the Creative Commons Attribution License, which permits unrestricted use, distribution, and reproduction in any medium, provided the original work is properly cited.

Preoperatively, the nodule was identified using cone beam computed tomography and fluoroscopy with the syngo iGuide and integrated needle guidance system (Siemens AG, Erlangen,

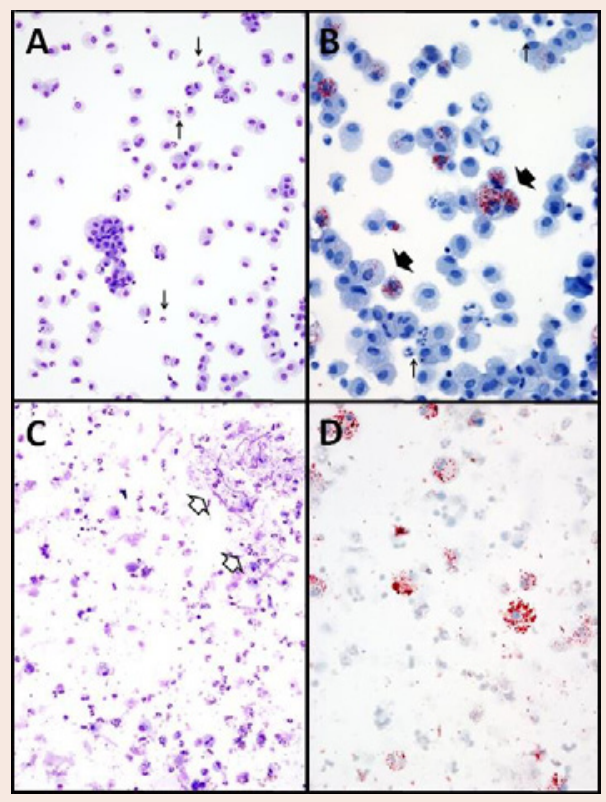

Figure 1: BAL specimens. Wright stain (A) Oil-Red-O stain (B) of original BAL specimen demonstrating a predominance of alveolar macrophages, including lipid-laden macrophages in low moderate numbers (thick arrows) and a low number of segmented neutrophils (thin arrows). There was no evidence of current or remote hemorrhage. (C, D) BAL specimen from one year later. Wright stain (C) Oil-Red-O stain (D) revealed an abnormal complement of an acute inflammatory infiltrate with degenerate cell debris, mucin (white arrow) and an abnormal complement of lipid laden macrophages. These findings were congruent with histology on the concurrent lung biopsy. 
Citation: Koehler SM, Heinzerling N, Moe DC, Gregg DC, Szabo S, et al. T-fasteners for Preoperative Localization of Pulmonary Nodules. J Pediatr Child Care. 2016;2(1): 5.

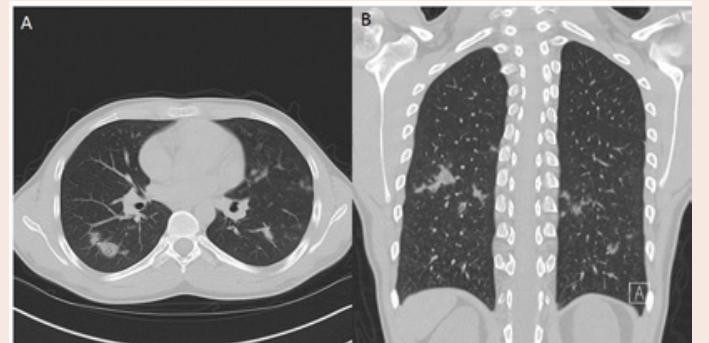

Figure 2: Preoperative CT Chest. (A) Axial and (B) coronal images showing several pulmonary nodules bilaterally.

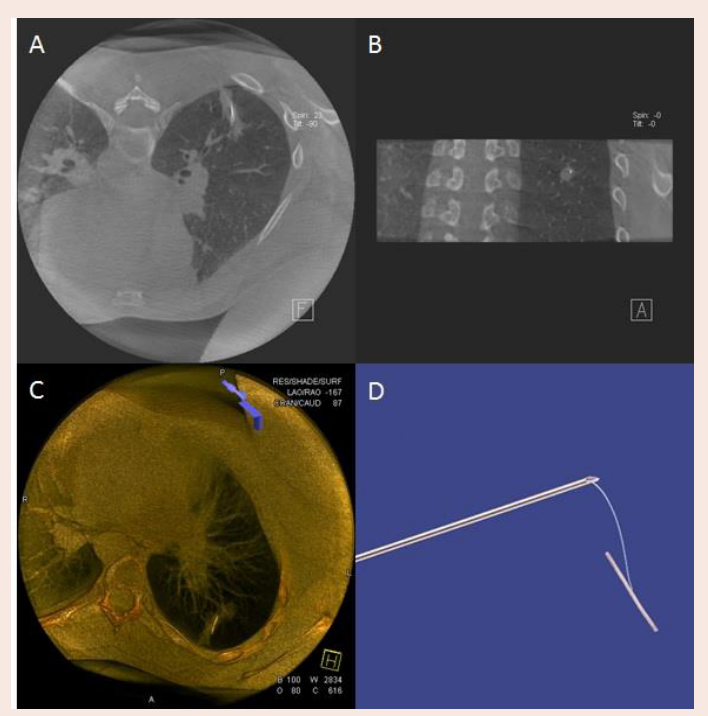

Figure 3: Images from the cone beam computed tomography guided $\mathrm{T}$-fastener placement. Images A and B show needle placement in the right lung. Image $\mathrm{C}$ shows volume rendered axial slab with T-fastener deployment. Image D shows a Cope gastrointestinal suture anchor. Orientation is reversed on these images.

Germany). Once the nodule was identified in the superior aspect of the right lower lobe of the lung, it was localized with a pediatric Cope gastrointestinal suture T-fastener (Cook Medical Inc., Bloomington, IL) and methylene blue (Figure 3). After deploying the T-fastener, the suture was brought out through the chest wall and secured to the skin with sterile gauze and adhesive dressing. The patient then underwent repeat flexible bronchoscopy with BAL followed by VATS. Singlelung ventilation was utilized, and when the right lung was dropped, the $\mathrm{T}$-fastener remained in position with the suture visible emanating from the lung parenchyma of the nodule in the superior aspect of the right lower lobe (Figure 4).

The patient tolerated the radiographic localization, bronchoscopy, and surgery well. The post-operative course was without incident and the patient was discharged on post-operative day three. The BAL fluid contained 4222 nucleated cells per microliter with $65 \%$ neutrophils, $13 \%$ lymphocytes and 22\% alveolar macrophages (Figures 1C and 1D). BAL cultures contained few Streptococcus viridans, few Neisseria species and few Staphylococcus species consistent with mixed oropharyngeal flora.
The biopsy specimen revealed a $0.7 \mathrm{~cm}$ yellow-white nodule surrounded by induration. Histology analysis was consistent with cryptogenic organizing pneumonitis with obstructing pneumonia in the surrounding lung parenchyma (Figure 5). No fungal mycelia, virus, or infective organism was present.

After the diagnosis of cryptogenic organizing pneumonia was obtained, the patient was started on systemic steroids for anticipated 6-month duration. Unfortunately, due to superimposed respiratory illnesses the patient has required a prolonged course of steroids with intermittent burst therapy. He is currently 2 years from his VATS biopsy and has been weaned off oral steroids.

\section{Cryptogenic Organizing Pneumonia}

\section{Pathophysiology}

Cryptogenic organizing pneumonia (COP), previously also known as "idiopathic bronchiolitis obliterans organizing pneumonia" (BOOP), is a diagnosis of exclusion. The pathophysiology of the disease is thought to be related to an abnormal response to lung injury. Typically, destruction of the airway epithelium is followed by inflammation and healing. This process goes awry in COP and the healing process is replaced by proliferation of granulation tissue. This leads to fibrosis of the airway and obliteration of the lumen [1].

\section{Etiology}

The disease process is typically idiopathic in nature. Associations have been made with connective tissue disorders, immunodeficiency syndromes, viral syndromes, bone marrow transplantation, graftversus-host disease and allograft rejection of lung transplants [2]. In addition, COP can also be seen after radiation exposure, with toxic exposures (including some medications) and may have seasonal variation $[2,3]$.

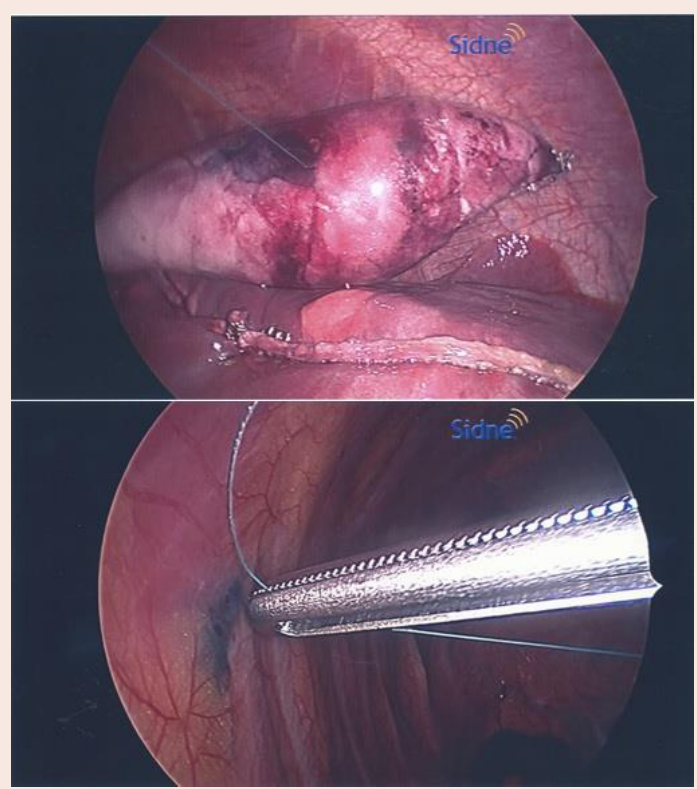

Figure 4: Intraoperative photos showing placement of the T-fastener in the lung and methylene blue staining of the pleural surface. The pleural surface is shown identifying the entry point of the T-fastener. 
Citation: Koehler SM, Heinzerling N, Moe DC, Gregg DC, Szabo S, et al. T-fasteners for Preoperative Localization of Pulmonary Nodules. J Pediatr Child Care. 2016;2(1): 5.

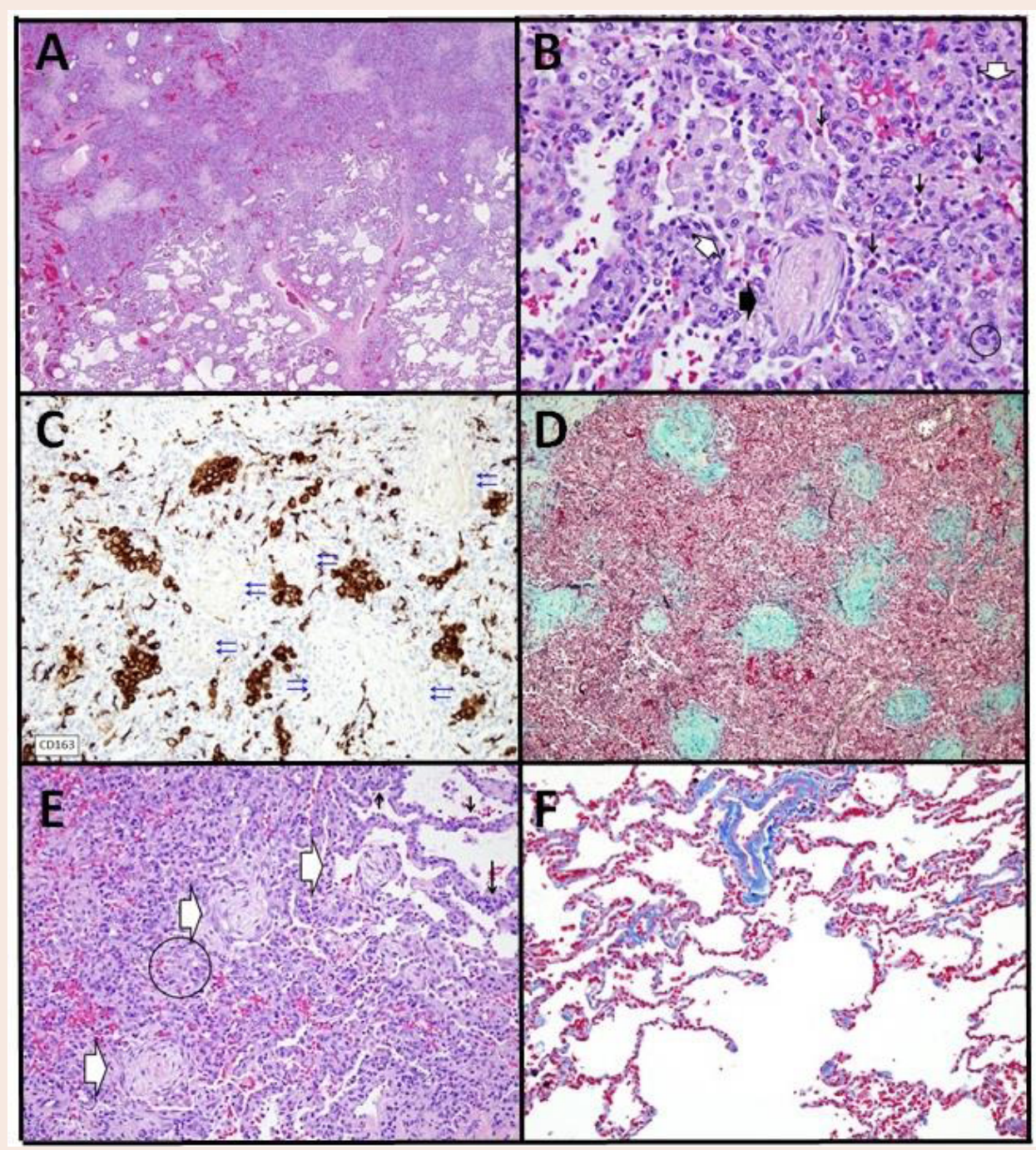

Figure 5: Histology on wedge resection consistent with cryptogenic organizing pneumonia. A) Interface between nodule with organizing pneumonia (upper half of image, solid cellular nodule with fibrous plugs), transitioning though an edematous zone, to unaffected lung parenchyma (right lower corner). B) The inflammatory infiltrate is predominantly mononuclear, including lymphocytes, plasma cells (circle), scattered macrophages, eosinophils (arrows) and sparse neutrophils, with collections of macrophages filling alveolar spaces (white arrow), and closely associated fibrosing nodules (black arrow). C) Entrapped alveolar macrophage plugs (highlighted on immunostain for CD163) alternate with paucicellular streaming fibrosing nodules and bands. Nuclei in the background stain (hematoxylin) include reactive alveolar lining and a mixed, predominantly mononuclear inflammatory infiltrate. D) In the lesional center, alveolar spaces are filled essentially completely with a cellular inflammatory infiltrate. Fibrous organizing nodules are highlighted on Movat pentachrome stain. E) At the lesional periphery, collapsed cellular lung parenchyma, with reactive alveolar septae (short thin black arrow) with a relatively mild inflammatory infiltrate, including plasma cells (long thin black arrow), alveolar macrophage plugs (circle), and fibrous organizing nodules protruding into alveoli (white thick arrows). F) In contrast, normal lung adjacent to the nodule (Trichrome stain).

\section{Presentation}

Patients typically present with nonspecific signs and symptoms. The majority of patients have fever, cough and dyspnea but other symptoms can include chest pain, weight loss and fatigue $[1,2]$. As the disease progresses, the patients have worsening hypoxemia and respiratory failure [1].

\section{Differential diagnosis}

The differential diagnosis for COP is wide. Other potential causes of multiple nodules on chest imaging and persistent cough can include infectious causes such as sarcoidosis, tuberculosis, mycoplasma pneumonia, aspergillus, several respiratory viral infections (especially in the pediatric population), septic emboli and other severe ischemic insults and infarcts, with subsequent organization [1,4]. Structural airway diseases, severe bronchiolitis and amyloidosis often present with chronic cough and numerous nodules on chest radiographs. Likewise, pneumoconiosis, such as silicosis, may produce similar signs and symptoms. Certain malignancies; such as nodular metastases, bronchoalveolar carcinoma, forms of pulmonary lymphoma, and kaposi sarcoma; may present in a similar manner to COP $[1,4]$. Yet, the disease most commonly confused with COP is chronic eosinophilic pneumonia (CEP). A simple blood eosinophil level is often adequate to distinguish these two entities as CEP will have a level $>1500 / \mathrm{mm}^{3}[1,5]$. 


\section{Diagnosis}

Chest CTs are an important step in the work up of patients with pulmonary abnormalities; however, the findings associated with COP are non-specific. The hallmark finding of COP on CT is patchy alveolar opacities [1]. These opacities can range in size from $1-2 \mathrm{~cm}$ and are often bilateral $[1,4,5]$.

Likewise, as seen with this patient, BAL is commonly used in the work up of COP. Cultures from BAL are generally only useful in ruling out other infectious causes of the patient's symptoms. However, analysis of the BAL fluid may suggest COP if there is a low CD4:CD8 ratio $(0.97 \pm 1.35)$ and elevated T lymphocytes (44.4 \pm 7.3$)$ as opposed $(1.65 \pm 1.71)$ and $(7.2 \pm 7.4)$ as is typically seen in usual interstitial pneumonia [6]. This patient's CD4:CD8 ratio was (0.6) and had $13 \% \mathrm{~T}$ lymphocytes further promoting the notion that BAL cannot be used to diagnose COP.

Histology is required for diagnosis of COP since signs, symptoms, imaging, bronchoscopy, BAL and pulmonary function testing are all nonspecific. VATS has been used since the late 1990s for diagnosis of interstitial lung disease after a paper demonstrated its safe and effective use in 49 patients (including 7 with COP) [7].

Once the biopsy is obtained, the diagnosis of COP is made by the histopathologic changes in the lung. This is characterized by patchy buds of granulation tissue in the connective tissue of the bronchioles, bronchiolar ducts and alveoli $[8,9]$.

\section{Treatment}

Prednisone is the first-line therapy of both symptomatic and progressive COP due to its potent anti-inflammatory properties. Treatment typically begins with $1 \mathrm{mg} / \mathrm{kg}$ for 1 to 3 months. Afterwards the patient is started on a prolonged taper of $40 \mathrm{mg} /$ day for 3 months then 10-20 mg every day or every other day for 6 months to one year. Total symptomatic and histologic recovery is typically obtained with this treatment regimen $[2,10]$. Recurrence of cryptogenic organizing pneumonia was found in up to one-third of patients treated for less than one year. However, recurrent episodes can be successfully treated with previously successful doses of prednisone. Importantly, in episodes of recurrent COP, the patient needs to be re-evaluated for an alternative diagnosis that could be causing the findings consistent with COP.

\section{Localization of the pulmonary nodule}

Numerous techniques exist for localization of solitary pulmonary nodules before open or thoracoscopic biopsy. The most widely techniques for localization are injection methylene blue at the site of the nodule or inserting a mammographic hook-wire adjacent to the nodule. However, each of these techniques is associated with failures or complications. Methylene blue is difficult to use in pulmonary tissue due to the inherent tendency to spread to the surrounding tissue especially in superficial nodules. Mammographic hook-wires are widely used for breast localization and have crossed over to localization of pulmonary nodules. However, they are associated with failure rates of up to $33 \%$ due to dislodgement of the needle from the pulmonary parenchyma or movement of the needle away from the desired nodule. Additionally, the placement of the needle is associated with a pneumothorax in $50-100 \%$ of cases [11].
Engeler described in vitro comparison of a hook-wire to T-fastener. In this study, they tested the weight needed to dislodge the hook-wire and T-fastener from formalin fixed and inflated cadaveric lungs and a synthetic sponge [11]. Interestingly, there was no significant difference in weight needed to dislodge either device from the lung and sponge. Additionally, they found no difference in friction of the wire or prolene suture as it traversed a $4 \mathrm{~cm}$ cadaveric chest wall.

Despite finding no strength advantage over the hook-wire, Engeler still hypothesized that the T-fastener has several theoretical advantages over the hook-wire. They note that the hook-wire is more likely to lacerate the pleura and cause a pneumothorax as the wire is under tension and less flexible compared to the prolene suture. Additionally, the T-fastener has a blunt tip and less likely to lacerate the lung or migrate compared to a hook-wire. Lastly, the suture outside the body is more manageable compared to the wire extruding from the body. This allows for easier transport and positioning of the patient pre-operatively.

The placement of the $\mathrm{T}$-fastener was achieved utilizing cone beam computed tomography (CBCT). CBCT is a relatively new technology that uses flat-panel detectors coupled together on a $\mathrm{C}$-arm. This allows for the acquisition of three-dimensional cross-sectional images in the interventional radiology suite without the need to transport the patient to a multi-detector computed tomography (MDCT) suite. These images are obtained by rotating the detector around the patient. The fundamental difference between CBCT and MDCT is that CBCT obtains information using a 2-dimensional detector while MDCT uses multiple single dimensional detectors. The major limitation to CBCT is that image resolution is inferior to MDCT and very small nodules may be difficult to visualize. CBCT has gained a wide berth of utility for hepatic embolization, partial splenic embolization, gastrostomy tube placement, skeletal procedures and biliary interventions. The immediate access to the patient afforded by CBCT as well as live fluoroscopy aids the anesthesiologist and interventional radiologist. In the event of a complication such as pneumothorax, not only can it be recognized sooner due to live fluoroscopy, it can increase the efficiency of chest tube placement.

\section{Conclusion}

VATS or open lung biopsy is vital to the diagnosis and management of lung nodules. Historically, localization of lung nodules has been challenging. Current management strategies utilize the injection of methylene blue around the nodule and inserting a hook-wire adjacent to the nodule. These techniques are associated with difficulties including displacement of the wire and spread of the blue dye to surrounding structures not associated with the nodule. Additionally, complications are associated with placement of a hookwire into the lung including injury to the lung and pneumothorax.

$\mathrm{T}$-fasteners are widely used for the placement of gastrostomy tubes $[12,13]$. More recently, the uses of T-fasteners has expanded and have been reportedly used for anterior chest wall elevation during a Nuss procedure [14], to prevent stent migration in tracheal obstruction [15], and to anchor a migrating inferior vena cava stent [16]. To our knowledge, this is the first published case utilizing a T-fastener for localization of a pulmonary nodule. The T-fastener offers the benefit 
Citation: Koehler SM, Heinzerling N, Moe DC, Gregg DC, Szabo S, et al. T-fasteners for Preoperative Localization of Pulmonary Nodules. J Pediatr Child Care. 2016;2(1): 5.

ISSN: 2380-0534

of (1) more precise localization than methylene blue; (2) more durable localization than the hook-wire as the potential for dislodgement is lessened with patient transport or single lung ventilation; and (3) it may offer safety advantages such as decreased risk of pulmonary laceration and subsequent pneumothorax. We found the T-fastener to be a highly valuable tool for pulmonary nodule localization preoperatively and believe its efficacy warrants further study.

\section{References}

1. Cottin V, Cordier JF (2012) Cryptogenic organizing pneumonia. Semin Respir Crit Care Med 33: 462-75.

2. Alasaly K, Muller N, Ostrow DN, Champion P, FitzGerald JM (1995) Cryptogenic organizing pneumonia. A report of 25 cases and a review of the literature. Medicine (Baltimore) 74: 201-211.

3. Ruth-Sahd LA, White KA (2009) Bronchiolitis obliterans organizing pneumonia. Dimens Crit Care Nurs 28: 204-208.

4. Ujita M, Renzoni EA, Veeraraghavan S, Wells AU, Hansell DM (2004) Organizing pneumonia: perilobular pattern at thin-section CT. Radiology 232 757-761.

5. Arakawa H, Kurihara $\mathrm{Y}$, Niimi H, Nakajima $\mathrm{Y}$, Johkoh $\mathrm{T}$, et al. (2001) Bronchiolitis obliterans with organizing pneumonia versus chronic eosinophilic pneumonia: high-resolution CT findings in 81 patients. AJR Am J Roentgenol 176: 1053-1058

6. Nagai S, Handa T, Ito Y, Takeuchi M, Izumi T (2007) Bronchoalveolar lavage in idiopathic interstitial lung diseases. Semin Respir Crit Care Med 28: 496503.

7. Deshmukh SP, Kransa MJ, McLaughlin JS (1996) Video assisted thoracoscopic biopsy for interstitial lung disease. Int Surg 81: 330-332.
8. (2000) American thoracic society. Idiopathic pulmonary fibrosis: diagnosis and treatment. International consensus statement. American thoracic society (ATS), and the European Respiratory Society (ERS). Am J Respir Crit Care Med 161(2 Pt 1): 646-664.

9. Pardo J, Panizo A, Sola I, Queipo F, Martinez-Penuela A, et al. (2013) Prognostic value of clinical, morphologic, and immunohistochemical factors in patients with bronchiolitis obliterans-organizing pneumonia. Hum Pathol 44: 718-724

10. Epler GR (2001) Bronchiolitis obliterans organizing pneumonia. Arch Intern Med 161: 158-164.

11. Engeler CE, Erickson PD, Tashijian JH, Hunter DW, Amplatz K, et al. (1994) Alternative design for radiologic guidance in video thoracoscopic surgery. Invest Radiol 29: 648-651.

12. Chadha KS, Thatikonda C, Schiff M, Nava H, Sitrin MD (2010) Outcomes of percutaneous endoscopic gastrostomy tube placement using a T-fastener gastropexy device in head and neck and esophageal cancer patients. Nutr Clin Pract 25: 658-662.

13. Thornton FJ, Fotheringham T, Haslam PJ, McGrath FP, Keeling F, et al (2002) Percutaneous radiologic gastrostomy with and without T-fastener gastropexy: a randomized comparison study. Cardiovasc Intervent Radiol 25: 467-471.

14. Kim D, Idowu O, Palmer B, Kim S (2014) Anterior chest wall elevation using a T-fastener suture technique during a Nuss procedure. Ann Thorac Surg 98: 734-736.

15. Musani Al, Jensen K, Mitchell JD, Weyant M, Garces K, et al. (2012) Nove use of a percutaneous endoscopic gastrostomy tube fastener for securing silicone tracheal stents in patients with benign proximal airway obstruction. $J$ Bronchology Interv Pulmonol 19: 121-125.

16. Wittich GR, Goodacre BW, Kennedy PT, Mathew P (2001) Anchoring a migrating inferior vena cava stent with use of a T-fastener. $\mathrm{J}$ Vasc Interv Radiol 12: 994-996. 